\title{
Dispersion anc Betatron Matching into the Linac
}

\author{
F.-J. Decker, C. Adolphsen, W.J. Corbett, P. Emma, \\ I. Hsu, H. Moshammer, J.T. Seeman, W.L. Spence, \\ Stanford Linear Accelerator Center; Stanford, Californio 94909
}

\section{Abstract}

In high energy linear colliders, the low emittanse beam from a damping ring has to be preserved all the way to the linac, in the linac and to the interaction point. In particular, the Ring-To-Linac (RTL) section of the SLAC Linear Collider (SLC) should provide an exact betatron and dispersion match from the damping ring to the linac. $A$ beam with a non-zero dispersion shows up immediately as an increased emittance, while with a betatron mismatch the beam filaments in the linac. Experimental tests and tuning procedures have shown that the linearized beta matching algorithms are insufficient if the actual transport line has some unknown errora not included in the model. Also, adjusting quadrupole strengths steers the beam if it is offset. in the quadrupole magnets. These and other effects have lead to a lengthy tuning process, which in the end improves the matehing, but is not optimal. Different ideas will be discussed which should improve this matching procedure and make it a more reliable, faster and simpler process.

\section{Theoretical Considerations}

A mismatch in betatron functions of the beam $(\alpha, \beta)$ and the lat tice $(\bar{\alpha}, \bar{\beta})$ and a non-zero dispersion $\left(\eta\right.$ or $\left.\eta^{\prime} \neq 0\right)$ at the beginning of the linac enlarges the effective emittance $\left(\epsilon_{e} / \rho\right)$. A dispersion $\eta$ causes different beam positions for different energies $\Delta x=\eta \Delta E / E$. This effect can be es timated by the following example. For an energy spread $\frac{\sigma_{E}}{E}=\delta \approx 1 \%$, a dispersion of $\eta=10 \mathrm{~mm}$ will lead to an emittance growth of roughly $10 \%$ (at a beam size of $\left.\sigma_{0}=\sqrt{c \beta}=316 \mu \mathrm{m}\right)$

$$
\epsilon_{e f f} \beta=\sigma^{2}=\epsilon \beta+\eta^{2} \delta^{2}=(0.1+0.01) \mathrm{mm}^{2},
$$

if there is a similar disturbance in the angular component with $\eta^{\prime}$. Otherwise the full expression has to be recognized:

$$
\epsilon_{e f}=\epsilon \sqrt{1+\left[\eta^{2}+\left(\beta \eta^{\prime}+\alpha \eta\right)^{2}\right]<\delta^{2}>/(\epsilon \beta)}
$$

which corresponds to a bigger (and additionally mismatched [1]) ellipse in phase space.

A betatron mismatch has no immediate effect on the emittance, but will increase the emittance by the filamentation of the phase space ellipse induced by an energy

"Work supported by the Department of Energy contract DEAC03-765Foosis. spread. This magnification due to the betatron mismatch is given by:

$$
\begin{gathered}
\beta_{\text {mag }}=\frac{1}{2}\left(\frac{\bar{\beta}}{\bar{\beta}}+\frac{\beta}{\bar{\beta}}\right)+\frac{1}{2}\left(\alpha \sqrt{\frac{\bar{\beta}}{\beta}}-\sqrt[a]{\frac{\beta}{\bar{\beta}}}\right)^{2} . \\
\text { e.E. } \alpha=\tilde{\alpha}=0 \begin{array}{cc}
\frac{(\beta-\bar{\beta}) / \bar{\beta}}{\beta_{\operatorname{mag}}-1} \\
\hline 0.5 & 0.08 \\
1.0 & 0.25 \\
2.0 & 0.67 \\
4.0 & 1.60 \\
n>10 & \approx n / 2
\end{array}
\end{gathered}
$$

Fig. 1 shows the beam in real space for $\beta$, $\eta$-mismatch and also higher order contributions. Besides the theoretical considerations, the observed practical problems during the actual minimization process will be described.

\section{Dispersion Match}

Although the dispersion adjustment should be performed only after the betatron mateh is done, it will be described first.

\subsection{Measurement}

There are two different techniques to determine the influence of the dispersion term:

1. The emittance, determind by wire seanners (or acreens), is compared for the two cases: a) with the normal $1 \%$ energy spread (compressor in RTL is on) and b) with no energy spread (compressor off).

2. By changing the phase and amplitude of the compressor to provide an acceleration, no acceleration or deceleration, it will lead to an offset $\Delta \mathbf{x}$ at the BPMs (beam position monitors), if dispersion term are present.

Additionally, a skewness in the beam distribution measured by a wire (sereen) indicates higher order energy dependencies ( $T_{166}[4]$ ), which can be determined by the BPM-data: For lower and higher energies $\Delta x$ has the same sign. 


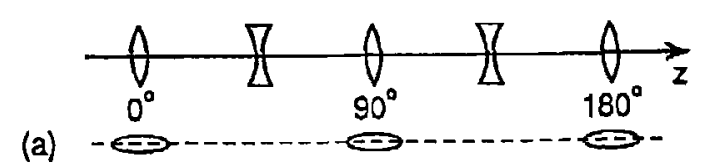

(b)

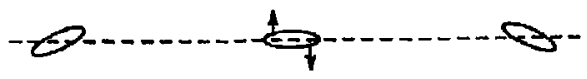

(c)

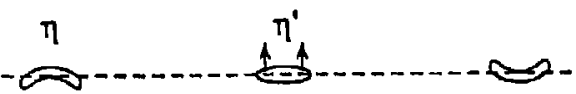

(d)

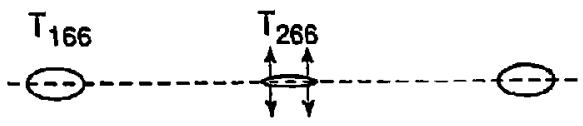

(e)

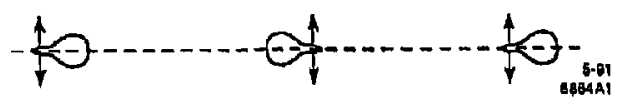

Figure 1: Beam Mism 2 thes.

The beam response in $x, z-s p a c e$ is shown for different mismatches. The beam has a langiludinal energy correlation introduced by a compressor and follows a $90^{\circ}$ per cell lallice (arrows indicate additional $x^{\prime}$ ): a) Matched beam, b) dispersion in space $\left(\eta=R_{16}\right)$ and angle $\left(\eta^{\prime}=R_{26}\right)$, c) higher order dispersion (or dispersive chromalicity): $T_{166}, T_{266}$, d) betatman mismatch $(\beta \approx 4 \beta)$, e) (betatron-) chromaticity is different focusing for different energies and resultant betatron match within the beam far not fully campressed bunches.

\subsection{Correction}

A combination of quadrupoles ( $\eta, \eta^{\prime}$-knobs) is changed, which should not influence the betatron match. Depending on the measurement techniques either the overall effect is decreased by minimizing the emittance or spot sizes, or the actual dispersion and higher orders are measured and can be compensated by a tight amount of the $\eta, \eta^{\prime}$-knobs and sextupole adjustments. Here some of the main techniques and their advantages are discussed:

1. The emittance is minimized and checked with wires at the beginning (or end) of the linac: The precise measurement leads to a more direct minimization of the important quantity, a residual dispersion may compensate rf-kicks and wakefields.

2. The two-dimensional spots on two screens are minimized by finding two good settings per sereen $\left(\eta, \eta^{\prime}\right)=$ $\left(\eta_{1}, 0\right)$ and $\left(0, \eta_{1}^{\prime}\right)$ and set to common solution: $I t$ is a quick method, but not necessarily the most accurate one.

3. Calculate and/or iterate BPM-data: The dispersion alone is minimized, residuals from rf-kick or wakefields remain. This method gives quantitative results for dispersion and also for higher orders.
For the higher order in dispersion, e.g. the betatron chromaticity, two methods have to be combined: At different energies, set by the compressor, the betat ron matching (see below) is measured with the wires.

\section{Betatron Match}

The twiss parameters $\alpha$ and $\beta$ of the beam have to be measured and compared to the design $\tilde{\alpha}, \tilde{\beta}$.

\subsection{Measurement}

The measurement is done by either a quadrupole scan or by multiple wire measurements. For the first method, the beam size on a screen or wire is recorded for different quadrupole settings. The second method needs at least three wires (screens), which measure the beam size at different phase advances giving $\alpha, \beta$ and $\epsilon$ (emittance) of the beam $[2,3]$. From the mismatch of the beam with respect to the design the beta-magnification $\beta_{\text {mag }}$ (see eq. 3 ) is calculated.

\subsection{Correction}

With the known lattice, $\alpha$ and $\beta$ of the beam can be tracked back to the beginning of the RTL and the corresponding beta-knobs changed by the appropriate amount. Most often the knobs are empirically tuned to minimize $\beta_{\text {mas. }}$.

\subsection{Difficulties with Betatron Adjustment}

A variety of problems, such as measurement inaccuracies, magnetic hysteresis and RTL focusing errors, makes the minimization of $\beta_{\text {mag }}$ below a value of 1.2 difficult. Some problems, their origin and their effects are summarized below:

1. Mieasurement error: Wire vibrations, photo multiplier saluration, bad timing of the gate (or nonlinearities of screens) may cause a wrong matching minimum.

2. Hysteresi, in magnets: $\beta_{x}, \beta_{y}$ knobs become nonorthogonal, especially if quadrupole trims have different signs in respect to common power supply.

3. Lattice: (i) Nearly degenerated matching knobs using only four quads require big changes, which steer the beam. (ii) Nonlinear knobs. (iii) Vertical focusing depends on steering through sextupoles.

4. Changing only one quad: Then less change is necessary. The measured hysteresis gap is $\approx 1 \%$, while a $0.6 \%$ change gives already $\beta_{\text {mag }}=2$ (see Fig. 2).

5. Not stable over days: With a different steering near the nonlinear septum or a vertical steering, the adjustment changes. 


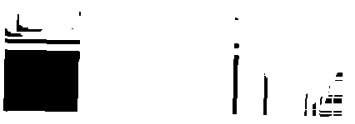

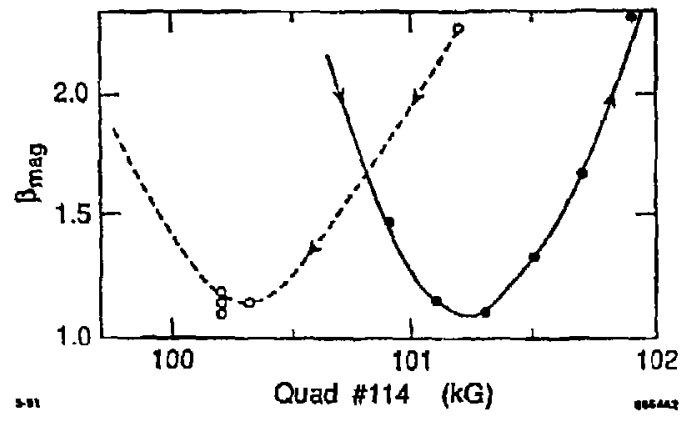

Figure 2: Betatron Match Sensitivity.

Bere one of the "matching" quadrupoles (number 114) in the RTL is changed by a few percent. For $0.4 \%$ change of the magnctic field the betatron magnification (corresponding to an emittance growih after filamentation) is 1.5. The difference going up or down the hysteresis loop (see orrows) is about $1 \%$ in field strength, which makes it difficult to adjust combinations of magnets.

\section{Precise Adjustment}

It should be mentioned that a coarse adjustment is achieved quite quickly and keeps the emittance blow-up below $30-50 \%$ of the $1.6 \cdot 10^{-5} \mathrm{mrad}$ normalized damping ring emittance. A precise adjustment can also be obtained, but usually only in one plane ( $x$ or $y$ ).

\subsection{Adjustment Procedures}

Over the years several betatron matcling "knobs" have been generated using four matehing quadrupoles. They are configured to change orthogonally one component of $\beta_{x}, \alpha_{x}, \beta_{y}, \alpha_{y}$ or more precisely, they contral the cosinelike, $\tilde{\beta} / \beta$, and sine-like, $\bar{\alpha}-\alpha \tilde{\beta} / \beta$, components of betabeat.

Historically the first set of krobs failed, since the power supply of one quad couldn't regulate well at the desired low amount. Then a second set was made using a small trim power supply for that quad. The difficulty with these knobs is that small misalignments of the strong matching quads cause a big steering of the beam. A third set uses only one matching quad and three pairs of quads in the dispersive region of the RTL. They require only small clianges (legs steering), but they have quite a large cross-talk between the $x$ and $y$ plane.

A different technique uses the four matching quads but with dynamic knobs. This means the measured response of the knobs is used to calculate the desired coefficjents for the four quads. A potential problem is the hysteresis of the magnets. Another method tries to avoid the hysteresis by calculating the desired clange and trimming the magnets to these values after standardization. This requires quite a long time, steers the beam and seens to bave a poor convergence.

The simplest way is taking only the most sensitive quad for the desired change and taking care of the normal standardization direction on the bystecesis loop. This is not totally orthogonal and therefore insufficient for a complete betatron match.

\subsection{New Ideas}

A number of ideas have been generated during discussions, but haven't been tried yet. One is to use more than four quads and minimizing the sum of changes to get the desired effect. This will be less degenerat, so less quad changes and therefore less beam steering will occur. Also the $z-y$ cross-talk might be less, but no hysteresis of the magnets are considered. To avoid hysteresis knobs may be used which alvays have the quads going in the standardization direction (or against for resetting). A variation might be a knob which brings a quadrupole current (if tweaked against hysteresis) far beyond the required value and then back following the standardization direction (quasi-standardized). Also a different standardization method [5] might be con* sidered, which is insensitive against tweaks around an optimal setting. Together with dynamic knobs, a fast procedure has to be found, since drifts of $20-30 \%$ in emittance over a few days have been observed.

\section{Conclusion}

Dispersion and betatron matching into the SLC-linac can reduce the emittance growth below about $30 \%$. For a further reduction, different procedures are needed, which deal with the problems of hysteresis in the magnets, measurement exrors, sensitivity of the lattice and long-term stabilily.

\section{References}

[1] N.Merminga, P.L.Morton, J.T.Seeman, W.L.Spence, Transverse Phase Space in the Presence of Dispersion, PAC, San Francisco, May 1991.

[2] M.C. Ross, N.Phinney, G.Quickfall, H.Shoaee, J.C.Sheppard, Automated Emitlance Measurement in the $S L C$, SLAC-Pub-4278 or PAC, Washington, D.C., March 1987.

[3] M.C. Ross, J.T.Seeman, E. Bong, L. Hendickson, D. MeCormick, L. Sanchez, Wire Scanners for Beam Size and Emittance Measurement at SLC, PAC, San Francisco, May 1991.

[4] C. Adolphsen, P.J. Emma, T.H. Fieguth, W.L. Spence, Chromatic Corretion in the SLC Bunch Length Compressor, PAC, San Mrancisco, May 1991.

[5] F.J. Decker, The Physica! Way of Standardizing Magnets, PAC, San Francisco, May 1991. 